\title{
SOC1 inhibits HPV-E7-mediated transformation by inducing degradation of $\mathbf{E} 7$ protein
}

\author{
Masaki Kamio ${ }^{1,2}$, Takafumi Yoshida ${ }^{1}$, Hisanobu Ogata ${ }^{1}$, Tsutomu Douchi ${ }^{2}$, Yukihiro Nagata ${ }^{2}$, \\ Makoto Inoue $^{3}$, Mamoru Hasegawa ${ }^{3}$, Yoshikazu Yonemitsu and Akihiko Yoshimura*,1 \\ ${ }^{1}$ Division of Molecular and Cellular Immunology, Medical Institute of Bioregulation, Kyushu University, 3-1-1 Maidashi, Higashi-ku, \\ Fukuoka 812-8582, Japan; ${ }^{2}$ Department of Obstetrics and Gynecology, Faculty of Medicine, Kagoshima University, 8-35-1 \\ Sakuragaoka, Kagoshima 890-0054, Japan; ${ }^{3}$ DNAVEC Research Inc. Tsukuba, Ibaraki, Japan; ${ }^{4}$ Division of Pathophysiological and \\ Experimental Pathology, Department of Pathology, Graduate School of Medical Sciences, Kyushu University 3-1-1 Maidashi, \\ Higashi-ku, Fukuoka 812-8582, Japan.
}

\begin{abstract}
Human papilloma viruses (HPVs) are small doublestranded DNA viruses that infect mucosal and cutaneous epithelium and induce cervical cancer. It has been shown that interferon (IFN) $\gamma$ suppresses proliferation of HPVinfected cells by suppressing expression of HPV E7. Here, we found that IFN $\gamma$ induces not only suppression of E7 transcription but also proteasome-dependent degradation. Suppressor of cytokine signaling-1 (SOCS1)/JAB, a suppressor of cytokine signaling, is known to be induced by IFN $\gamma$, and functions as an antioncogene against various hematopoietic oncogenic proteins. SOCS1 contains the SOCS-box, which is shown to recruit ubiquitin transferase to the molecules that interact with SOCS1. We found that SOCS1 interacted with HPV E7 protein and induced ubiquitination and degradation of E7 in a SOCS-boxdependent manner. SOCS1 overexpression also increased Rb protein levels and suppressed proliferation of cervical cancer cell lines infected with HPV. Moreover, E7 protein levels were higher and $\mathbf{R b}$ protein levels were lower in SOCS1-deficient fibroblasts infected with retrovirus vector carrying E7 gene than in wild-type fibroblasts. E7 induced anchorage-independent growth in SOCS1-deficient fibroblasts, but not in wild-type cells. These data suggested that SOCS1 plays an important role in regulating the levels of $\mathrm{E} 7$ protein and their transforming potential, and could be a new therapeutic tool for HPVmediated tumors.
\end{abstract}

Oncogene (2004) 23, 3107-3115. doi:10.1038/sj.onc. 1207453 Published online 15 March 2004

Keywords: cytokine; SOCS; papilloma viruses; interferon gamma; E7; proteasome

\section{Introduction}

Suppressor of cytokine signaling-1 (SOCS1) is originally identified as a JAK tyrosine kinase binding protein; we

*Correspondence: A Yoshimura;

E-mail: yakihiko@bioreg.kyushu-u.ac.jp

Received 21 October 2003; revised 7 December 2003; accepted 9 December 2003 and others have shown that SOCS1 inhibits many cytokine-signaling pathways by inhibiting JAK tyrosine kinase activity. SOCS1 belong to a family called CIS/ SOCS family that is composed of eight family members. Most of them are induced by cytokines and believed to be a negative feedback regulator of cytokine signaling. However, recent studies suggest that SOCS1 regulates not only cytokines but also c-kit, Vav, IRS-1 and other signaling pathways (De Sepulveda et al., 1999, 2000; Kawazoe et al., 2001; Ilangumaran et al., 2003). Especially, SOCS1 is suggested to function as antioncogene. Yoshikawa et al. (2001) reported aberrant methylation in the $\mathrm{CpG}$ island of SOCS1 that correlated with its transcription silencing in HCC cell lines. The incidence of aberrant methylation was $65 \%$ in the 26 human primary HCC tumor samples analysed. Moreover, the restoration of SOCS1 suppressed both the growth rate and the anchorage-independent growth of cells in which SOCS1 was methylation-silenced, and JAK2 was constitutively activated. Recently, SOCS1 methylation was also reported in human hepatoblastomas and multiple myeloma (Galm et al., 2003; Nagai et al., 2003). Experimentally, Rottapel et al. (2002) showed that SOCS1-deficient fibroblasts were more sensitive to both spontaneous or oncogene (v-ABL, p210 BCR-ABL or 70Z/3 CBL)-induced transformation than wild-type fibroblasts, indicating that SOCS1 functions as an antioncogene. However, the precise molecular mechanism of the antioncogenic activity of SOCS1 is not fully understood.

SOCS1 binds directly to the conserved regulatory tyrosine in the activation loop of the JAK2 kinase (JH1) domain through its $\mathrm{SH} 2$ domain and inhibits JAK kinase activity through occlusion of the substrate binding site (Nicholson et al., 1999; Yasukawa et al., 1999). However, suppression of the oncogenic activity of TEL-JAK2, a constitutive active form of JAK 2 tyrosine kinase, required not only kinase inhibition but also SOCS-box-mediated ubiquitination and degradation of TEL-JAK2. SOCS1 also binds to and destabilizes the guanine nucleotide exchange factor VAV-1 (De Sepulveda et al., 2000), an important c-kit substrate. SOCS1 binds to VAV though the SH2 domain of SOCS1, but in a phosphotyrosine-independent manner. It also forms 
a physical complex with components of the VCB-type E3 ubiquitin ligase, Elongin $\mathrm{B}$ and $\mathrm{C}$, through its Cterminal SOCS-Box, which recruits Cul-2, Rbx-1 and E2 ubiquitin transferase complex (Kamura et al., 1998; Zhang et al., 1999). Therefore, SOCS1 is now believed to function as an adapter molecule to recruit target proteins such as VAV and TEL-JAK2 for ubiquitination and subsequent degradation (Zhang et al., 1999; De Sepulveda et al., 2000; Kamizono et al., 2001).

Human papilloma virus (HPV) is a major cause of cervical cancer (Bosch et al., 1995). The high-risk HPVs (such as HPV-16 and HPV-18) encode two oncoproteins, E6 and E7 (Munger and Howley, 2002). HPV E7 induces transformation of rodent fibroblast cell lines (Phelps et al., 1988), and also contributes to the transformation of primary human cells (Hawley-Nelson et al., 1989; Munger et al., 1989). The E6 and E7 genes of the high-risk HPV types are necessary and sufficient for the efficient immortalization of primary squamous epithelial cells (Munger et al., 1989; Watanabe et al., 1989). Whereas E6/E7 antisense constructs are also able to selectively inhibit growth of cervical carcinoma cells expressing these genes (Tan and Ting, 1995; von Knebel Doeberitz et al., 1988), indicating that E6 and E7 are necessary for the maintenance of transformed phenotypes. It has been reported that E7 is short lived (Selvey et al., 1994) and is regulated by the ubiquitin-proteasome pathway in cervical cancer cells (Reinstein et al., 2000; Wang et al., 2001). However, an E3-ubiquitin ligase for E7 has not been identified.

We report here that ectopic expression of SOCS1 abolished proliferation of cervical cancer cell lines by inducing destabilization of E7. SOCS1 directly interacts with E7 and promotes its ubiquitination in a SOCS-boxdependent manner. Moreover, SOCS1-deficient fibroblasts were sensitive to E7-mediated cell proliferation and transformation. These data indicate that SOCS1 is a candidate as an E3-ubiquitin ligase for E7, and that SOCS1 could be a new therapeutic target for cervical cancer treatments.

\section{Results}

Interferon (IFN) $\gamma$ induced expression of SOCSI and degradation of E7 in cervical cancer cell lines

The treatment of HPV-associated diseases with IFNs has undergone many clinical trials. A compilation of data from various studies indicates that generally IFN $\gamma$ is more effective than type 1 IFNs, although the reason for this has not been clarified. It has been reported that IFN $\gamma$ reduces E7 transcription (Kim et al., 2000). We noticed that treatment of the cervical cancer cell lines, $\mathrm{HeLa}$ and $\mathrm{CaSki}$, with IFN $\gamma$ resulted in the reduction of E7 protein levels (Figure 1a). Although E7 mRNA levels determined by RT-PCR were reduced $72 \mathrm{~h}$ after IFN $\gamma$ treatment in Hela and CaSki cells (Figure 1b), this reduction of proteins levels has not been correlated with the decrease in the E7 mRNA levels. Thus, we hypothesized that IFN $\gamma$ induces $\mathrm{E} 7$ protein level

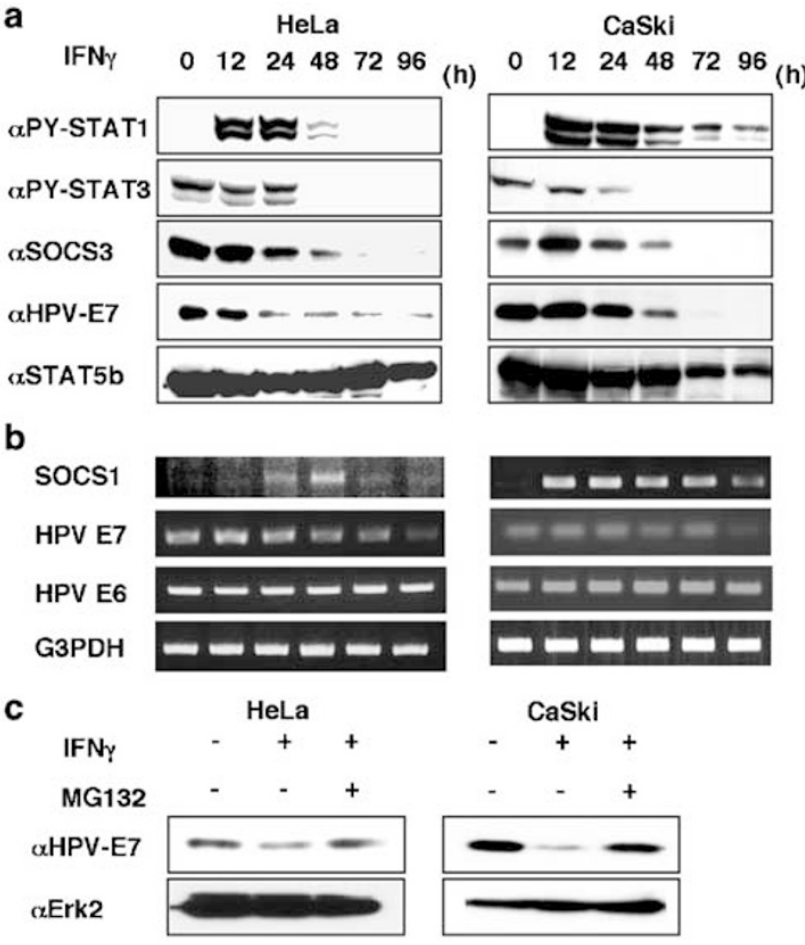

Figure 1 IFN $\gamma$ induced expression of SOCS1 and degradation HPV-E7 in cervical cancer cell lines. (a) HeLa and CaSki cell were stimulated with $100 \mathrm{U} / \mathrm{ml} \mathrm{IFN} \gamma$ for the indicated periods. After the indicated time, total cell extracts was blotted with anti-phosphoSTAT1, anti-phospho-STAT3, anti-SOCS3, anti-HPV-E7 and anti-STAT5b. (b) After the indicated time, total RNA was extracted. RT-PCR analysis was performed to detect SOCS1, HPV-E7, HPV-E6 and G3PDH. (c) CaSki and HeLa cells were stimulated with $100 \mathrm{U} / \mathrm{ml} \mathrm{IFN} \gamma$ for $72 \mathrm{~h}$ and $100 \mu \mathrm{M}$ MG132 for $4 \mathrm{~h}$. Total cell extract was blotted with anti-HPV16-E7 and Erk2

degradation. This is supported by the fact that the proteasome inhibitor MG132 inhibits IFN $\gamma$-mediated reduction of E7 proteins (Figure 1c).

IFN $\gamma$ is a potent inducer of SOCS1, which contains the SOCS-box that might recruit ubiquitin transferase E2 to the interacting target molecules. As shown in Figure $1 \mathrm{~b}$, IFN $\gamma$ strongly induced SOCS1 mRNA in HeLa and CaSki cells, and when the SOCS1 levels reach a plateau at $12-48 \mathrm{~h}$ of IFN $\gamma$ treatment, E7 protein decreased drastically.

\section{Constitutive expression of SOCS1 inhibits the proliferation of cervical cancer cell lines}

We examined the effect of the forced expression of SOCS1 on the function of E7 in HeLa and CaSki cells. First, SOCS1 was overexpressed in these cells using a Sendai virus vector $(\mathrm{SeV})$ carrying the SOCS1 gene. The recently developed $\mathrm{SeV}$ possesses very high infection efficiency, like an adenovirus vector, and the expression of the carrying gene persists much longer than with an adenovirus (Yonemitsu et al., 2000). Overexpression of SOCS1 reduced proliferation rate in both HeLa and CaSki cell lines (Figure 2a). Suppression of DNA synthesis by SOCS1 was confirmed by reduced incorporation of $\mathrm{BrdU}$ (data not shown). To avoid the 

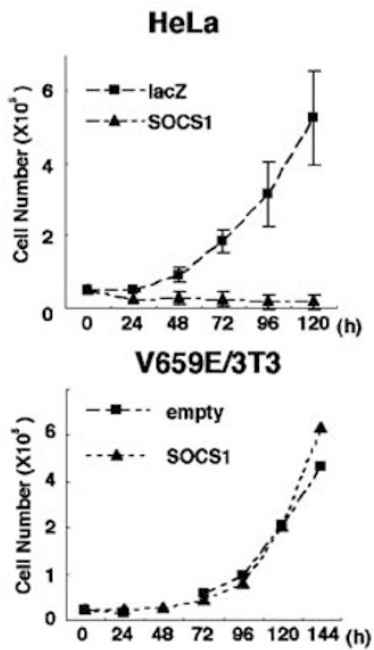

b

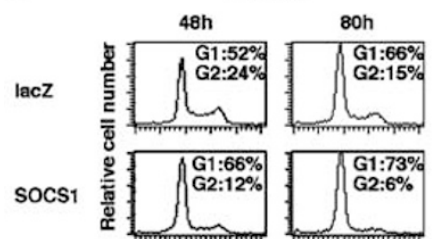

C

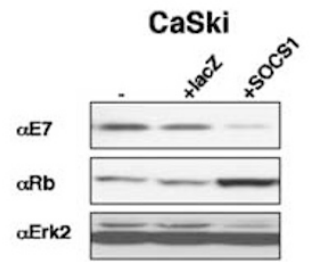

Figure 2 SOCS1 and SOCS3 inhibit the proliferation of cervical cancer cell lines. (a) HeLa and CaSki cells were infected with a Sendai virus carrying the SOCS1 and lacZ, then plated into $35 \mathrm{~mm}$ dishes and cell numbers were counted daily under normal growth conditions. Data represent mean + s.d. of triplicate measurements. NIH3T3 cells transformed with Erb-B2 were infected with SOCS1, then the cell number was counted daily under normal growth conditions. (b) $\mathrm{HeLa}$ and CaSki cells were infected with Sendai virus. Then, the cells were harvested at various time points postinfection, resuspended in PI solution $(500 \mu \mathrm{g} / \mathrm{ml}$ PI, $0.1 \%$ Triton X-100, $0.1 \%$ sodium citrate), and then samples were subjected to FACS. The G1 and the G2 phase populations are shown. (c) CaSki cells were infected with Sendai virus carrying SOCS1 and lacZ at MOI $=1$. At $80 \mathrm{~h}$ after infection, total cell extracts were blotted with anti-Rb, anti-E7 and Erk2

possibility that SOCS1 is a general inhibitor of cell proliferation, we examined the effect of NIH3T3 cells transformed with Erb-B2. Proliferation of these cells was not affected by SOCS1 overexpression (Figure 2a). To clarify the molecular mechanism of the reduced proliferation caused by SOCS1, the cell cycle profile was analysed by flow cytometry. Apoptotic cells (detected in propidium iodide (PI)-low fraction) were not increased by SOCS1 infection, whereas G1 arrest was observed by SOCS1 overexpression (Figure 2b). Thus, forced expression of SOCS1 did not affect apoptosis, but induced cell cycle arrest. It has been shown that E7 induces downregulation of $\mathrm{Rb}$ proteins, which may contribute to
Table 1 Tumor size in SCID mice

\begin{tabular}{llr}
\hline Cell line & Tumor size $(\mathrm{mm})$ & Volume $\left(\mathrm{mm}^{3}\right)$ \\
\hline Hela & $14 \times 9 \times 8$ & 1008 \\
& $12 \times 7 \times 4$ & 336 \\
Hela/lacZ & $24 \times 15 \times 16$ & 4680 \\
& $12 \times 9 \times 9$ & 972 \\
Hela/SOCS1 & $10 \times 8 \times 8$ & 640 \\
& $8 \times 4 \times 3$ & 96 \\
& 0 & 0 \\
& 0 & 0 \\
& $2 \times 2 \times 1$ & 0 \\
\hline
\end{tabular}

HeLa cells $\left(1 \times 10^{7}\right.$ cells $)$ infected with Sendai virus at MOI $=0.5$ were injected subcutaneously into the flanks of SCID mice. Tumor growths were monitored for 3 weeks. Tumor size was determined by the product of two perpendicular diameters and the height above the skin surface.

cell proliferation and transformation (Munger and Howley, 2002). Interestingly, SOCS1 overexpression resulted in the reduction of E7 protein levels and in the increase in $\mathrm{pRb}$ levels (Figure 2c). SOCS1-mediated cell cycle arrest may be explained by upregulation of $\mathrm{pRb}$ levels.

SOCS1 also strongly inhibited tumorigenicity of HeLa cells when cells were injected into SCID mice (Table 1). These data indicate that SOCS1 overexpression suppresses proliferation and tumorigenicity of HPV-infected cell lines.

\section{Effect of SOCS1 on STAT3 activation}

To further investigate the molecular mechanism of SOCS1-mediated suppression of cervical cancer cells, we examined STAT3 activation, since constitutively activated STAT3 plays an important role in transformation, and SOCS1 is believed to be a strong inhibitor of the JAK/STAT pathway. As shown in Figures 1a and 3a, HeLa cells showed constitutive activation of STAT3. Ectopic overexpression of the dominant negative STAT3 (dnSTAT3), SOCS3 and SOCS1 by adenovirus vector infection completely diminished STAT3 phosphorylation (Figure 3a). However, both SOCS3 and dnSTAT3 only partially suppressed HeLa cell proliferation, whereas SOCS1 completely inhibited HeLa cell proliferation (Figure $3 b$ ). These data suggest that the antiproliferative effect of SOCS1 may be partly dependent on STAT3, but an independent mechanism is present.

\section{SOCS1 promotes degradation of HPV E7}

Proliferation of cervical cancer cells is shown to profoundly depend on E7 and E6 viral oncoproteins (Venturini et al., 1999). Therefore, we examined the effect of SOCS1 on E7 protein levels based on the fact that IFN $\gamma$ induces SOCS1 and E7 degradation. As shown in Figure 4a, ectopic expression of SOCS1 downregulates HPV E7 protein levels dose dependently in HeLa and CaSki cells, but does not alter E7 mRNA levels. This decrease in E7 protein levels by SOCS1 was 


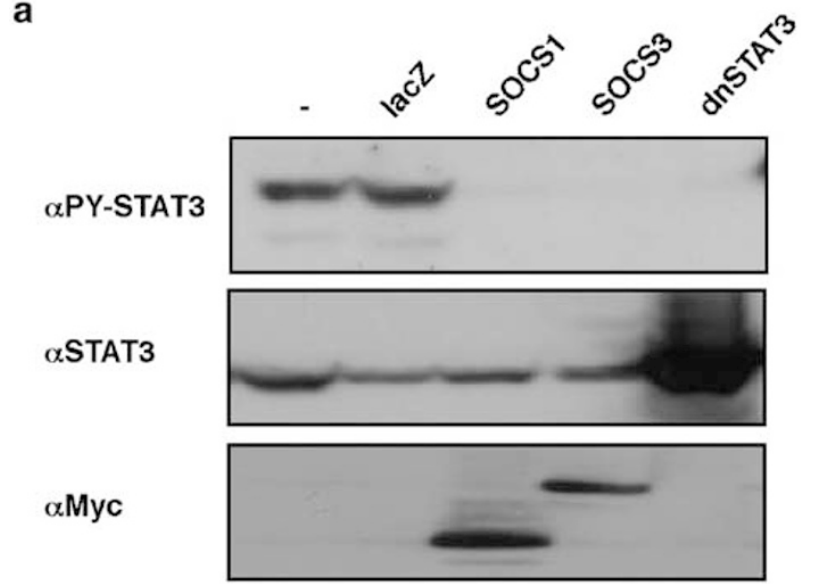

b

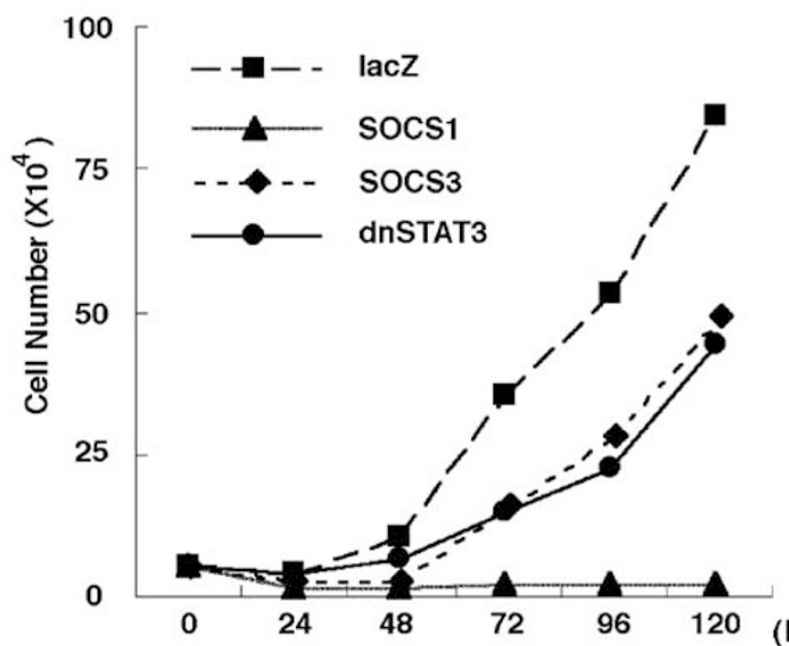

Figure 3 SOCS3 and dominant-negative (dn) STAT3 abolish STAT3 phosphorylation, but only partially suppress HeLa cell growth. (a) HeLa cells were infected with adenovirus (at MOI =5) carrying SOCS1, SOCS3, dnSTAT3 and lacZ. Total cell extracts were blotted with indicated antibodies. (b) HeLa cells were infected with adenovirus (at MOI $=5$ ) carrying SOCS1, SOCS3, dominantnegative STAT3 and lacZ. Cell numbers were counted daily under normal growth conditions

prevented by the proteasome inhibitor MG132, suggesting that SOCS1 promotes ubiquitination and degradation of E7 molecules.

To further investigate this effect, we transiently expressed E7 with SOCS1 in HEK293 cells and observed E7 protein expression levels. As shown in Figure 4b, E7 protein levels decreased as SOCS1 protein levels increased. The half-life of E7 molecules was significantly shortened by SOCS1 overexpression (Figure 4c). This SOCS1-mediated degradation of E7 protein was strongly inhibited by MG132. We also examined the effect of SOCS3, which was also expressed in Hela and CaSki cells (Figure 1a). However, SOCS3 did not induced degradation of E7 protein (Figure 4c), which is consistent with partial inhibitory effect of SOCS3 on Hela cell proliferation (Figure $3 b$ ). These data raise a possibility that SOCS1 is a specific ubiquitin ligase for E7.

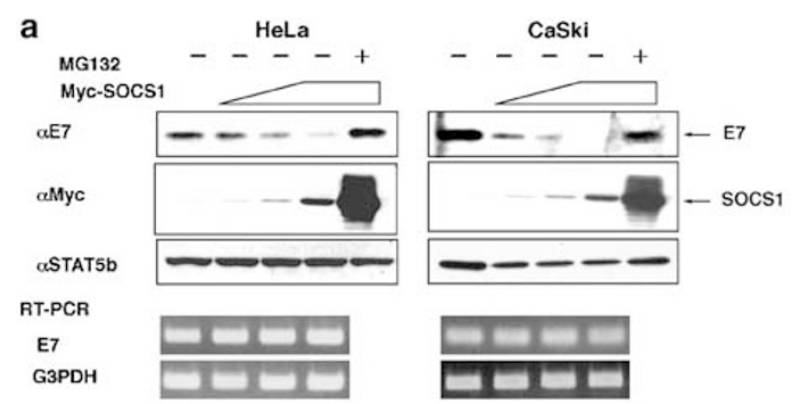

b

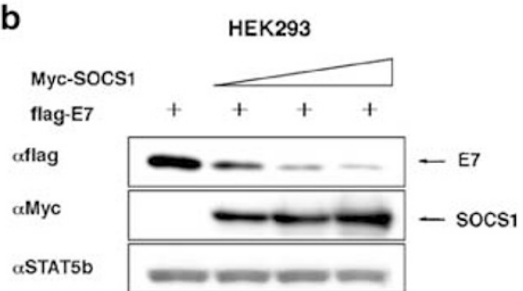

C

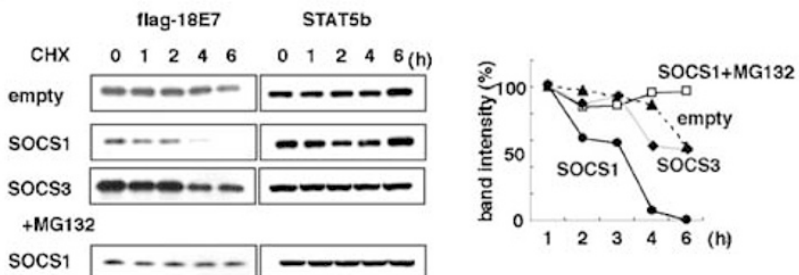

Figure 4 SOCS1 promotes degradation of HPV E7. (a) HeLa cells were transfected with $0,0.3,0.5,1.0$ and $1.0 \mu \mathrm{g}$ plasmid of pcDNA3-Myc-SOCS1 and then cultured. At $24 \mathrm{~h}$ after transfection, cells were treated with $100 \mu \mathrm{M}$ MG132 for $4 \mathrm{~h}$. Total cell extracts were blotted with anti-HPV18-E7, anti-Myc, antiSTAT5b. Total RNA was extracted from cells, then RT-PCR was performed to detect HPV18-E7 and G3PDH. CaSki cells were transfected with $0,0.05,0.1,0.5$ and $0.5 \mu \mathrm{g}$ plasmid of pcDNA3Myc-SOCS1 and then cultured. Total cell extracts were blotted with anti-HPV16-E7, anti-Myc, anti-STAT5b. Total RNA was extracted from cells, then RT-PCR was performed to detect HPV16-E7 and G3PDH. (b) HEK293 cells were transiently cotransfected with p3XFLAGCMV10-18E7 $1.0 \mu \mathrm{g}$ with $0,0.1$, $1.0,1.5 \mu \mathrm{g}$ pcDNA3-Myc-SOCS1. Total cell extracts were blotted with anti-flag, anti-Myc and anti-STAT5b. (c) HEK293 cells were transiently cotransfected with p3XFLAGCMV10-18E7 $1.0 \mu \mathrm{g}$ with $1.0 \mu \mathrm{g}$ pcDNA3-empty vector and pcDNA3-Myc-SOCS1 or pcDNA3-Myc-SOCS3. $24 \mathrm{~h}$ after transfection, cells were treated with $100 \mu \mathrm{g} / \mathrm{ml}$ cycloheximide (CHX) and $100 \mu \mathrm{M} \mathrm{MG132}$ (the bottom lane) for the indicated time. Total cell extracts were blotted with anti-flag and anti-STAT5b. The levels of flag-tagged E7 were quantified by densitometry of the immunoblots using NIH image software

\section{SOCS1 interacts with E7 and induces ubiquitination}

To confirm our hypothesis, we examined the interaction between SOCS1 and E7. Immunoprecipitation assay revealed that SOCS1 directly interacted with E7 molecule (Figure 5a). E7 bound to recombinant GSTSOCS1 in vitro (Figure 5b). Using deletion mutants, we found that SOCS1 interacted with E7 through the SH2 domain region, since dN77 that contains the $\mathrm{SH} 2$ domain and the SOCS-box still bound to E7, but the 


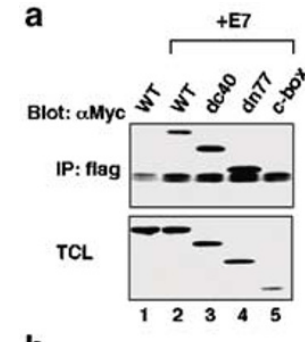

b
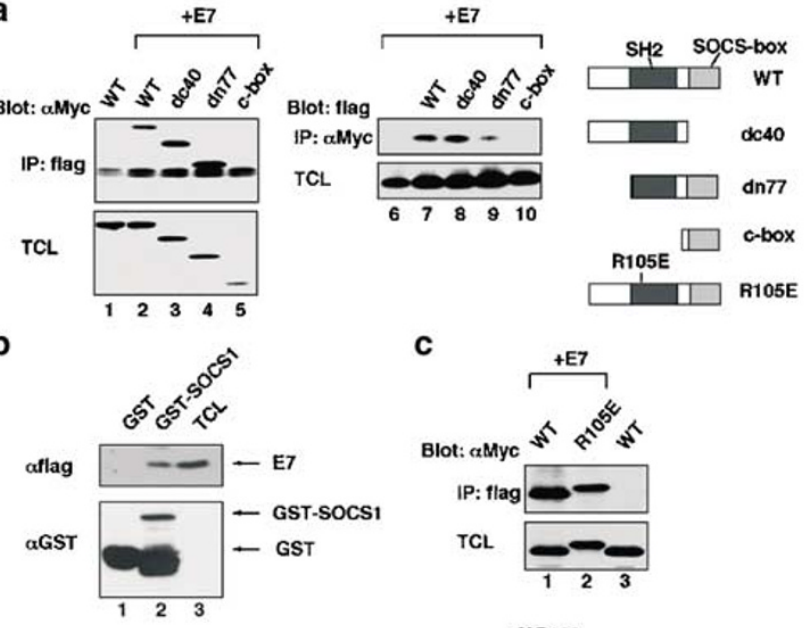

C

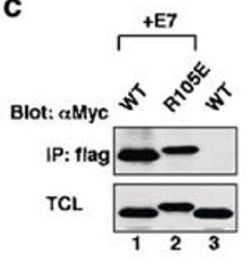

d

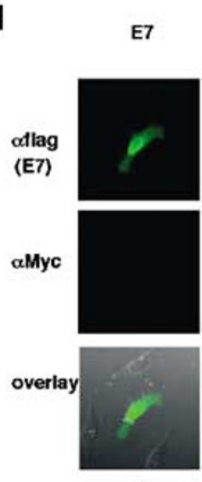

E7
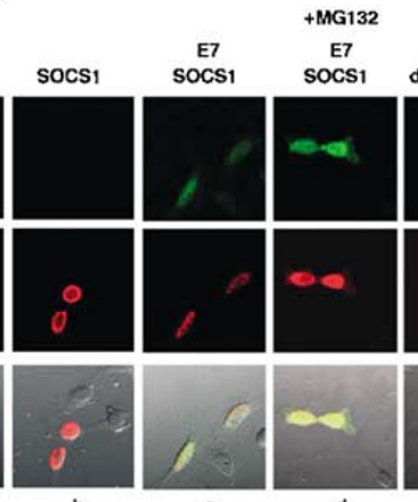

d

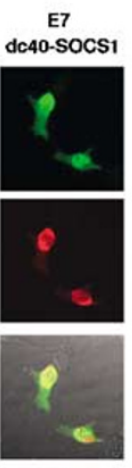

e

Figure 5 Colocalization of HPV E7 and SOCS1. (a) HEK293 cells were transiently transfected with p3XFLAGCMV10-18E7 (lanes 210), pcDNA3-empty vector (lane 6), pcDNA3-Myc-SOCS1 (lanes 1,2,7), pcDNA3-Myc-dC40-SOCS1 (lanes 3,8), pcDNA3-MycdN77-SOCS1 (lanes 4,9) and pcDNA3-Myc- c-box-SOCS1 (lanes 5,10). A schematic structure of the mutants is shown. The immunoprecipitates with anti-flag antibody and anti-Myc antibody were analysed with anti-Myc and anti-flag, respectively. (b) In vitro binding experiment. HEK 293 cells were transiently transfected with p3XFLAGCMV10-18E7. Total cell extracts were incubated with either GST or GST-SOCS1 bound to glutathione-Sepharose. Aliquots of total cell extract (TCL) and proteins bound to the beads were immunoblotted with anti-flag and anti-GST. (c) HEK293 cells were transiently cotransfected with p3XFLAGCMV10-18E7 together with pcDNA3-Myc-SOCS1 or pcDNA3Myc-R105E-SOCS1. The immunoprecipitates with anti-flag antibody and anti-Myc antibody were analysed with anti-Myc and anti-flag, respectively. R105E SOCS1 mutant migrate slowly in SDS-PAGE probably because of drastic change of the charge (Yasukawa et al., 1999). (d) HeLa cells were transfected with p3XFLAGCMV10-18E7 or cotransfected with p3XFLAGCMV10-18E7 and pcDNA3-Myc-SOCS1 or dC40-SOCS1. At $24 \mathrm{~h}$ after transfection, cells were treated with $100 \mu \mathrm{M}$ MG132 for $2 \mathrm{~h}$ (lane d), then fixed and stained with anti-flag (polyclonal; green) or anti-Myc (monoclonal; red) antibodies followed by incubation with FITC and Alexa Fluor-conjugated secondary antibodies

SOCS-box alone (C-box) did not bind to E7 (Figure 5a). However, R105E mutant, which disrupts the SH2 domain function, still bound to E7 (Figure 5c). Therefore, the SOCS1-E7 interaction seems to be independent of tyrosine phosphorylation of E7, which is similar to

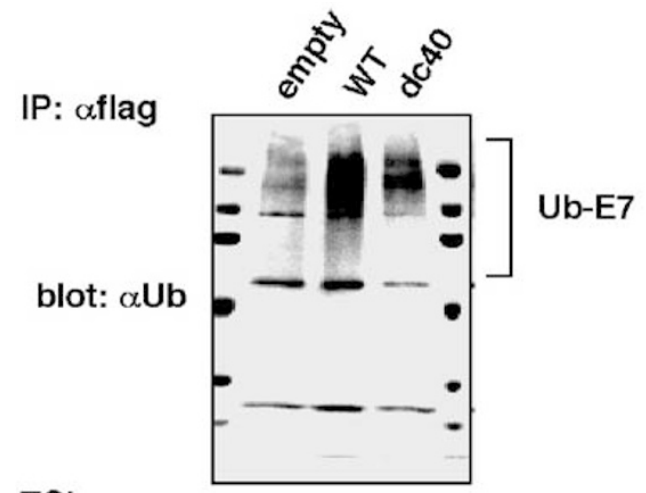

TCL

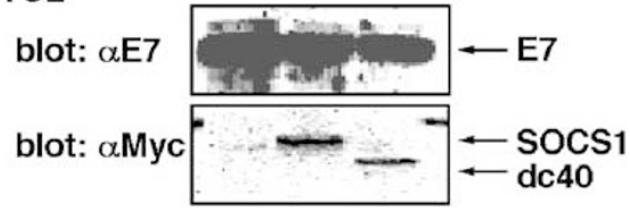

Figure 6 SOCS1 promotes degradation of E7 through the proteasome pathway. (a) HEK293 cells were transiently cotransfected with $0.5 \mu \mathrm{g}$ p3XFLAGCMV10-18E7 with $2.0 \mu \mathrm{g}$ pcDNA3empty vector, pcDNA3-Myc-SOCS1 and pcDNA3-Myc-dC40SOCS1. At $12 \mathrm{~h}$ after transfection, cells were treated with $20 \mu \mathrm{M}$ MG132 for $1 \mathrm{~h}$. Total cell extracts were blotted with anti-ubiquitin (Ub)

the interaction of SOCS1 with Vav (De Sepulveda et al., 2000).

Next, to confirm interaction between SOCS1 and E7 in cells, we examined the intracellular localization of SOCS1 and E7 by immunostaining (Figure 5d). E7 is shown to be present in both cytoplasm and nucleus without SOCS1 (Figure 5d-a). Interestingly, we found that SOCS1 is mostly accumulated in the nucleus (Figure 5d-b). When SOCS1 and E7 were coexpressed, they were mostly colocalized in the nucleus (Figure 5dc), suggesting that E7 was accumulated in the nucleus with SOCS1, or cytoplasmic E7 and SOCS1 were degraded very rapidly. To address these possibilities, we examined the effect of MG132. In the presence of MG132, both SOCS1 and E7 were accumulated not only in the nucleus but also in the cytoplasm (Figure 5dd). These data suggest that SOCS1 interacts with E7 in the nucleus and is degraded rapidly by the proteasome in the cytoplasm. DC40 mutant SOCS1 lacking the SOCS-box was present both in the cytoplasm and nucleus (Figure 5d-e), like SOCS1 treated with MG132 (Figure 5d-d). Thus, SOCS-box-dependent degradation may occur in the cytoplasm.

Then we examined the ubiquitination of E7 in vivo. Flag-E7 was coexpressed with Myc-tagged WT or dC40-SOCS1 lacking the SOCS-box, then immunoprecipitated with anti-flag antibody and blotted with anti-ubiquitin antibody (anti-Ub). As shown in Figure 6, WT-SOCS1 promoted ubiquitination of E7, whereas dC40-SOCS1 promoted less accelerated E7 ubiquitination. These data indicate that SOCS1 promotes ubiquitination and degradation of E7 in a SOCS-box-dependent manner. Thus, SOCS1 function as an ubiquitin ligase for E7. 


\section{SOCS1-deficient fibroblasts are more sensitive to E7-mediated transformation}

To investigate the physiological role of SOCS1 on E7mediated transformation, we used SOCS1-deficient embryonic fibroblasts. Populations of embryonic fibroblasts were derived from either wild-type or SOCS1-1day 15 embryos. We infected these cells with retrovirus carrying E7 and GFP. It has been shown that overexpression of E7 caused proteolytic degradation of the tumor suppressor $\mathrm{Rb}$ (Wang et al., 2001). As shown in Figure $7 \mathrm{a}$, the levels of E7 protein were higher in SOCS1 $1^{-1-}$ fibroblasts than in $\mathrm{SOCS}^{+/+}$fibroblasts, although E7 mRNA levels were similar. GFP protein

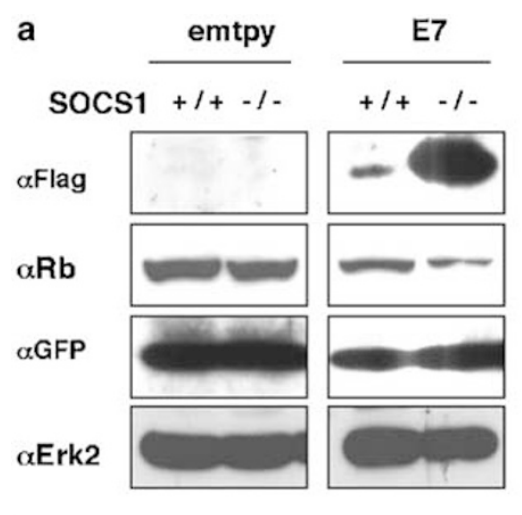

\section{b}

E7

G3PDH

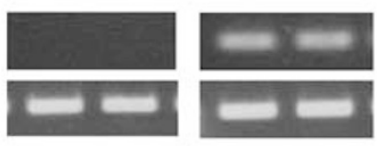

C
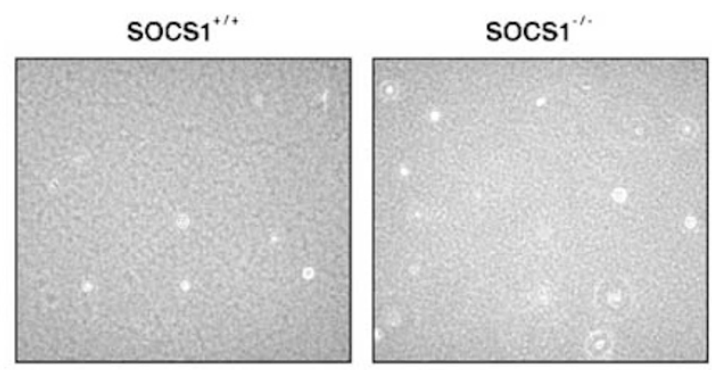

E7
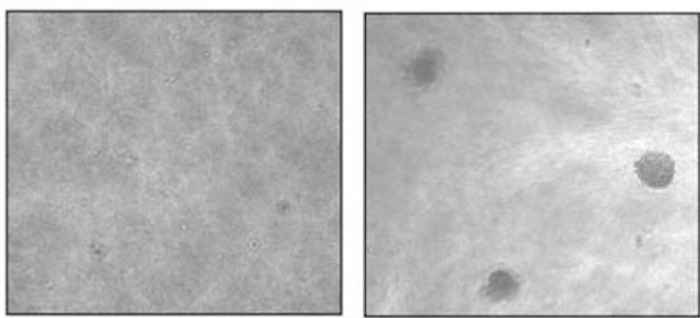

Figure 7 Transforming potential of HPV E7 in SOCS1-deficient fibroblasts. Populations of embryonic fibroblasts were derived from $\mathrm{SOCS}^{+/+}$or SOCS1 $1^{-/-}$day 15 embryos. These cells were infected with retrovirus carrying Flag-E7. Over $80 \%$ infection was confirmed by EGFP fluorescence. (a) Cells were expanded for 1 week, then total cell extracts were blotted with indicated antibodies. (b) $\mathrm{SOCS}^{+/+}$and $\mathrm{SOCS}^{-/-}$embryonic fibroblasts were infected with retrovirus carrying HPV16 E7 and empty vector. Cells were expanded for 2 weeks, then plated into soft agar and photographed after 3 weeks levels that derived from the E7-IRES-GFP mRNA were similar between $\mathrm{SOCS}^{+/+}$and $\mathrm{SOCS}^{-/-}$cells, further indicating that the mRNA levels of E7 were similar. In contrast, $\mathrm{Rb}$ protein levels were decreased by E7 expression, and were further downregulated in SOCS1deficient fibroblasts expressing E7. While it has been shown that E7 alone cannot induce cellular transformation, as depicted in Figure 7b, E7 induced colony formation of SOCS1 ${ }^{-/-}$fibroblasts, but not SOCS1 ${ }^{+/+}$ fibroblasts in soft agar. These data indicate that a SOCS1 deficiency potentiates the oncogenic potential of E7 in fibroblasts.

\section{Discussion}

The process of cell transformation includes both the activation of oncogenes and the suppression of inhibitory signals that normally operate to regulate the duration and intensity of signals. As a negative regulator of cytokine signaling, SOCS1 is shown to be a candidate gene for suppressing hematopoietic oncogenes including v-Abl, Bcr-Abl and TEL-JAK2 (Rottapel et al., 2002). Although molecular mechanism for these antioncogenic activity of SOCS1 has not been completely understood, it is notable that SOCS1 could induce ubiquitination and degradation of interacting proteins in a phosphotyrosine-dependent and -independent manner (De Sepulveda et al., 2000).

HPV-E7 functions as an oncogene by interacting with hypophosphorylated members of the $\mathrm{pRb}$ family for ubiquitin-mediated proteolysis, resulting in decreased $\mathrm{pRb}$ levels and inappropriate releases of active $\mathrm{E} 2 \mathrm{~F}$ transcription factor in cells (Wu et al., 1993; Jones and Munger, 1996). However, E7 is a 'sticky' protein; it interacts with a large number of different proteins (Munger et al., 2001). Moreover, E7 is unstable protein and is itself ubiquitinated. Thus, if a protein possessing a potential of ubiquitin transferase activity binds to E7, it could be an E3 ubiquitin ligase for E7. As shown in the present study, SOCS1 can interact phosphotyrosine independently with E7 through the $\mathrm{SH} 2$ domain region. Therefore, it is probable that SOCS1 overexpression can promote ubiquitination and degradation of E7, thereby suppressing proliferation and tumorigenicity mediated by E7. Indeed, as shown in Figure 6, SOCS1 promoted ubiquitination of E7. However, since dC40 mutant that lacked the SOCS-box still induced weak ubiquitination of E7 (Figure 6), involvement of other ubiquitin ligases may be involved. Binding of $\mathrm{dC} 40$ to E7 could modify the structure of E7, which is more accessible to another ubiquitin ligase for E7, or SOCS1 N-terminal region may form a homo- or heterooligomer with other ubiquitin ligases. Therefore, dC40 may recruit another ubiquitin ligase to E7. In any case, E3 ubiquitin ligases against E7, including SOCS1, could be new therapeutic targets for treatment of HPVmediated tumors.

The E7 protein has been shown to localize mainly in the nucleus, although a substantial fraction of the E7 
protein is found in cytoplasm. We also found that SOCS1 is highly accumulated in the nucleus. This is very interesting because SOCS1 has been thought to inhibit JAK kinases by direct interaction, and JAKs are present in cytoplasm or plasma membrane. We found that SOCS1 as well as E7 protein accumulated in cytoplasm when cells were treated with MG132. This suggests that SOCS1 and E7 were ubiquitinated and degraded in cytoplasm but not in the nucleus. Therefore, like p27 cdk inhibitor (Tomoda et al., 2002), the mechanism of export of SOCS1 and E7 may be very important for maintenance of the levels of these proteins. We could not find any nuclear export motifs in SOCS1 and E7, thus mutational analysis will be necessary for definition of the nuclear export signals. Importantly, dC40-SOCS1 is mostly present in the nucleus (Kamio et al., unpublished data). This indicates that the SOCS-box may be necessary not only for the ubiquitination of SOCS1 itself but also for nuclear export of SOCS1.

Extensive surveys of primary tumors and cell lines derived from tumors have indicated that constitutive activation of STATs, especially STAT3, occurs at a surprisingly high frequency in a wide variety of human cancers (Yoshida et al., 2002). First, we thought that the inhibitory effect of SOCS1 against the growth of HeLa and CaSki cells was due to the suppression of STAT3 activation. However, as shown in Figure 3, dnSTAT3 that inhibits STAT3 activation, only partially prevented cell proliferation. Therefore, STAT3 activation might not be a major factor in the proliferation of these cells. Our data are consistent with the notion that E7 and E6 are the most important proteins for transformed phenotypes of cervical cancer cells. E7 protein has been shown to target $\mathrm{pRb}$ to ubiquitin-mediated proteolysis, resulting in decreased pRb levels (Wu et al., 1993; Jones and Munger, 1996). While the mechanism of the reduction of $\mathrm{pRb}$ by E7 has not been clarified, one possible mechanism is ubiquitination of $\mathrm{pRb}$ by the E7-SOCS1 complex. In the present study, we showed that overexpression of SOCS1 upregulated $\mathrm{Rb}$ levels, while loss of SOCS1 gene resulted in further decreases of $\mathrm{Rb}$. Further study is necessary to clarify the physiological E3 ubiquitin ligase for $\mathrm{pRb}$, but inhibition of E7-mediated $\mathrm{pRb}$ degradation by SOCS1 could account for the antioncogenic activity of the SOCS1 gene (Rottapel et al., 2002).

IFNs have antiviral, immunomodulatory, and antiproliferative activities (Kalvakolanu and Borden, 1996; Stark et al., 1998). Previous report have shown that IFN $\gamma$ inhibits proliferation of the cervical cancer cell lines, HeLa and CaSki (Song and Shuai, 1998; Um et al., 2000). Consistent with the result of Woodworth et al, we showed that in both cell lines the protein expression levels of HPV E7 were reduced with IFN $\gamma$ treatment (Woodworth et al., 1992). This downregulation of E7 protein appears to occur not only at the transcriptional level but also at the post-transcriptional level, since proteasome inhibitor prevented the reduction of E7 proteins levels induced by IFN $\gamma$ (Figure 1c). Our study suggests that SOCS1 induced by IFN $\gamma$ is, at least in part, responsible for the reduction of E7 and suppression of proliferation. At a clinical level, IFNs have been used in the treatment of HPV-associated cervical intraepithelial neoplasia or cervical cancers with mixed results (Gross, 1997; Koromilas et al., 2001). The success of the treatment is largely dependent on the subtype of HPV and the immune responses of the patients. Since SOCS1 gene expression is epigenetically regulated by gene methylation (Yoshikawa et al., 2001), differences in the levels of SOCS1 induced by IFN $\gamma$ in individuals could account for the different levels of sensitivity in patients. Our study raises the possibility that induction of SOCS1 is a new therapeutic way for treatment of HPV-mediated cervical cancers.

\section{Materials and methods}

\section{Cell culture}

The human cervical cancer cell lines, HeLa and CaSki, were obtained from the Japan Health Sciences Foundation and maintained in DMEM and RPMI1640 containing $10 \%$ fetal calf serum (FCS), respectively. HEK293 and NIH3T3 were cultured in DMEM containing 10\% calf serum (CS). Primary cultures of embryonic fibroblasts (MEF) were derived from day $15 \mathrm{SOCS}^{-/-}$and wild-type mouse embryos obtained by crossing $\mathrm{SOCS}^{+/}$male and female mice. Cells were maintained in DMEM containing $10 \%$ FCS. MEFs were analysed within passage number 20 .

\section{Construction of recombinant $\mathrm{SeV}$}

Recombinant SeVs were constructed, as described (Kato et al., 1996; Sakai et al., 1999; Yonemitsu et al., 2000). In brief, 18 bp of spacer sequence 5'-(G)-CGGCCGCAGATCTTCACG-3' with a NotI restriction site (underlined) were inserted between the $5^{\prime}$ nontranslated region and the initiation codon of the nucleoprotein $(\mathrm{N})$ gene. This cloned $\mathrm{SeV}$ genome also contains a self-cleaving ribosome site from the antigenomic strand of the hepatitis delta virus. The entire cDNA coding SOCS-1, SOCS-3 and lacZ were amplified by PCR, using their specific primers with a NotI site and new sets of $\mathrm{SeV} \mathrm{E}$ and $\mathrm{S}$ signal sequence tags for an exogenous gene, and then inserted into the NotI site of the cloned genome. The entire length of the template $\mathrm{SeV}$ genome, including exogenous genes, was arranged in multiples of six nucleotides (so-called 'rule of six') (Kolakofsky et al., 1998). Template SeV genome with an exogenous gene and plasmids encoding $\mathrm{N}, \mathrm{P}$ and $\mathrm{L}$ genes (plasmid pGEM-N, pGEM-P and pGEM-L, respectively) were complexed with commercially available cationic lipids, then cotransfected with UV-inactivated vaccinia virus vT7-3 into CV-1 or LLMCK cells (Fuerst et al., 1986). After $40 \mathrm{~h}$, the cells were disrupted by three cycles of freezing and thawing, and then injected into the chorioallantoic cavity of 10-day-old embryonated chicken eggs. Subsequently, the virus was recovered and the vaccinia virus was eliminated by a second propagation in eggs. Virus titer was determined by hemagglutination assay using chicken red blood cells (Yonemitsu et al., 1998), and the virus then stored at $-80^{\circ} \mathrm{C}$ until use.

\section{Other vectors and plasmids}

Adenoviral vectors containing the genes for LacZ, Myc-tagged SOCS1, SOCS3 and dominant-negative STAT3 (dnSTAT3) were described previously Shouda et al. (2001). SOCS1 and deletion mutants in pcDNA3 expression vector were described 
(Yasukawa et al., 1999). Deletion mutant Myc-c-box-SOCS1, which contains only the SOCS-box of SOCS1 (codon 167-2-12), was also generated by standard PCR methods as described previously. The HPV18-E7 and 16E7 cDNA was subcloned into a bicistronic retrovirus vector, pMX-IRES-EGFP (enhanced green fluorescent protein) (Nosaka et al., 1999; Sasaki et al., 2000).

Preparation of a retrovirus and infection to MEF have been described (Sasaki et al., 2000). The HPV18 E7 region (318 nucleotides) and HPV16 E7 region (297 nucleotides) were amplified by RT-PCR using HeLa and CaSki cell cDNAs as the template, respectively. The nucleotide and amino-acid sequence of HPV16 E7 completely match those of the HPV strain PPH16. The nucleotide and amino-acid sequences of HPV18 E7 are 99\% identical to those of the HPV strain PAPHPV18. These cDNAs were subcloned into Flag-tagged expression vector p3XFLAGCMV10 (Sigma). Transient transfections of plasmids were carried out using CellPhect (Amersham Biosciences) for 293 cells and PolyPhcet (QIAGEN) for HeLa and CaSki cells.

\section{Proliferation assays}

Cells were seeded at $5 \times 10^{5} /$ well in $10 \mathrm{~cm}$ dishes, then infected with $\mathrm{SeV}$ at an MOI of 2 for HeLa cells and an MOI of 1 for CaSki cells. After $24 \mathrm{~h}$, cells were removed by trypsinization and seeded onto six-well plates at $5 \times 10^{4}$ cells/well. Cell numbers were counted every $24 \mathrm{~h}$.

\section{SCID mice xenograft experiments}

Male C. B-17/IcrCrj-SCID mice (6 weeks old) were purchased form Charles River Laboratories. HeLa cells $\left(1 \times 10^{7}\right.$ cells $)$ infected with $\mathrm{SeV}$ at an MOI of 0.5 were injected subcutaneously into the flanks of SCID mice. Tumor growths were monitored weekly for 3 weeks. Tumor size was determined by the product of two perpendicular diameters and the height above the skin surface.

\section{Colony assay}

SOCS1 $1^{+/-}$and ${ }^{-/-}$fibroblasts were infected with retrovirus carrying HPV16-E7 or vector alone, and cells were seeded into $35-\mathrm{mm}$ dishes in suspensions of $0.36 \%$. Agar noble (Difco) in DMEM supplemented with $10 \%$ FCS on top of a bed of $0.72 \%$ Agar noble in the same complete medium. The cultures were incubated for 21 days and then colonies were counted and photographed.

\section{Immunohistochemistry}

Cells were plated on chamber slides and transfected with HPV18 E7 and SOCS1 mutants. At $24 \mathrm{~h}$ after transfection, $100 \mu \mathrm{M}$ MG132 was added for $2 \mathrm{~h}$. The cells were fixed with PBS containing $4 \%$ paraformaldehyde for $10 \mathrm{~min}$, then rendered permeable with PBS containing $0.1 \% \mathrm{NP}-40$ for $5 \mathrm{~min}$ at room temperature. After blocking nonspecific antibodies binding sites with $10 \%$ goat serum, the cells were incubated with a rabbit anti-flag (Affinity Bioreagents) and mouse anti-Myc (SantaCruz) antibodies. Bound antibodies were detected with a goat

\section{References}

Bosch FX, Manos MM, Munoz N, Sherman M, Jansen AM, Peto J, Schiffman MH, Moreno V, Kurman R and Shah KV. (1995). J. Natl. Cancer Inst., 87, 796-802.

De Sepulveda P, Ilangumaran S and Rottapel R. (2000). J. Biol. Chem., 275, 14005-14008. anti-rabbit secondary antibody conjugated to FITC (ZYMED Laboratories) and a goat anti-mouse secondary antibody conjugated to Alexa Fluor (Molecular Probes) and viewed under a confocal microscope (Carl Zeiss).

\section{FACS analysis}

HeLa and CaSki cells were seeded at $1 \times 10^{5} /$ well in $3.5 \mathrm{~cm}$ dishes and incubated for $12 \mathrm{~h}$. Cells were infected with $\mathrm{SeV}$ (at MOI $=2$ and $\mathrm{MOI}=1$, respectively). Then cells were harvested at various time points postinfection, centrifuged at 1500 r.p.m. for $3 \mathrm{~min}$, washed in PBS, and the pellet resuspended in $500 \mu \mathrm{l}$ of PI solution $(500 \mu \mathrm{g} / \mathrm{ml}$ PI, $0.1 \%$ Triton X-100, $0.1 \%$ sodium citrate). After incubation for $30 \mathrm{~min}$ at room temperature, samples were subjected to FACS.

\section{$R T-P C R$}

Total cell RNA was extracted using Trizol reagent as per the manufacture's instructions (Life Technologies, Australia). The primer sets used for the semiquantitative PCR were: for HPV18 E7: 5'-atgcatggacctaaggcaacattgcaagac- $3^{\prime}$ and 5'ttactgctgggatgcacaccacggacacac-3'; for HPV18 E6: 5'-atgctg catgccataaatgtatagatttt- $3^{\prime}$ and $5^{\prime}$-ttatacttgtgtttctctgcgtcgttg gag-3'; for HPV16 E7: 5'-GCAACCAGAGACAACTGATC$3^{\prime}$ and $5^{\prime}$-ATTGTAATGGGCTCTGTCCG-3'; for HPV 16E6: $5^{\prime}$-AAGGGCGTAACCGAAATCGGT-3' and $5^{\prime}$-GGAATC TTTGCTTTTTGTCC- $3^{\prime}$; for SOCS 1 : $5^{\prime}$-cacgcacttccgcacattcc3' and 5'-tccagcagctcgaagaggca $-3^{\prime}$; for G3PDH: 5'-ACCA CAGTCCATGCCATCAC- $3^{\prime}$ and $5^{\prime}$-TCCACCACCCTGTT GCTGTA-3'

\section{Immunoprecipitation and Western blot analysis}

Immunoprecipitation and immunoblotting were performed as described previously (Kamizono et al., 2001). For the cycloheximide treatment experiment, HEK293 cells were transfected with E7 with WT or mutant SOCS1. At $18 \mathrm{~h}$ after transfection, cells were incubated with $50 \mu \mathrm{g} / \mathrm{ml}$ cycloheximide for the indicated periods and immunoblotted with anti-E7 and anti-Myc antibodies. Anti-HPV18 E7 goat polyclonal antibody, anti-HPV16 E7 monoclonal antibody, anti-STAT5b, anti-ERK2, anti-Myc, anti-GFP, anti-GST were purchased from Santa-Cruz Biotechnology; anti-tyrosine-phosphorylated STAT1 and anti-tyrosine-phosphorylated STAT3 were from Cell Signaling Technology; anti-flag M2 was from SIGMA; anti-pRb was from Pharmingen. For anti-ubiquitin blotting, transferred membranes were treated with $0.5 \%$ glutaldehyde at room temperature for $30 \mathrm{~min}$ as described (Jeon et al., 2002).

\section{Acknowledgements}

We thank Ms Y Kawabata-Honda and Ms S Sata for excellent technical assistance, Ms N Arifuku and Ms F Yamaura for manuscript preparation. This work was supported by Special Grants-in-Aid from the Ministry of Education, Science, Technology, Sports and Culture of Japan, Mochida Memorial Foundation, and the Uehara Memorial Foundation.
De Sepulveda P, Okkenhaug K, Rose JL, Hawley RG, Dubreuil $\mathrm{P}$ and Rottapel R. (1999). EMBO J., 18, 904-915

Fuerst TR, Niles EG, Studier FW and Moss B. (1986). Proc. Natl. Acad. Sci. USA, 83, 8122-8126. 
Galm O, Yoshikawa H, Esteller M, Osieka R and Herman JG. (2003). Blood, 101, 2784-2788.

Gross G. (1997). Intervirology, 40, 368-377.

Hawley-Nelson P, Vousden KH, Hubbert NL, Lowy DR and Schiller JT. (1989). EMBO J., 8, 3905-3910.

Ilangumaran S, Finan D, Raine J and Ilangumaran R. (2003). J. Biol. Chem., 278, 41871-41880.

Jeon JH, Cho SY, Kim CW, Shin DM, Kwon JC, Choi KH and Kim IG. (2002). Exp. Mol. Med., 34, 496-499.

Jones DL and Munger K. (1996). Semin. Cancer Biol., 7, 327-337.

Kalvakolanu DV and Borden EC. (1996). Cancer Invest., 14, 25-53.

Kamizono S, Hanada T, Yasukawa H, Minoguchi S, Kato R, Minoguchi M, Hattori K, Hatakeyama S, Yada M, Morita S, Kitamura T, Kato H, Nakayama $\mathrm{K}$ and Yoshimura A. (2001). J. Biol. Chem., 276, 12530-12538.

Kamura T, Sato S, Haque D, Liu L, Kaelin Jr WG, Conaway RC and Conaway JW. (1998). Genes Dev., 12, 3872-3881.

Kato A, Sakai Y, Shioda T, Kondo T, Nakanishi M and Nagai Y. (1996). Genes Cells, 1, 569-579.

Kawazoe Y, Naka T, Fujimoto M, Kohzaki H, Morita Y, Narazaki M, Okumura K, Saitoh H, Nakagawa R, Uchiyama Y, Akira S and Kishimoto T. (2001). J. Exp. Med., 193, 263-269.

Kim KY, Blatt L and Taylor MW. (2000). J. Gen. Virol., 81, 695-700.

Kolakofsky D, Pelet T, Garcin D, Hausmann S, Curran J and Roux L. (1998). J. Virol., 72, 891-899.

Koromilas AE, Li S and Matlashewski G. (2001). Cytokine Growth Factor Rev., 12, 157-170.

Munger K, Basile JR, Duensing S, Eichten A, Gonzalez SL, Grace M and Zacny VL. (2001). Oncogene, 20, 7888-7898.

Munger K and Howley PM. (2002). Virus Res., 89, 213-228.

Munger K, Phelps WC, Bubb V, Howley PM and Schlegel R. (1989). J. Virol., 63, 4417-4421.

Nagai H, Naka T, Terada Y, Komazaki T, Yabe A, Jin E, Kawanami O, Kishimoto T, Konishi N, Nakamura M, Kobayashi Y and Emi M. (2003). J. Hum. Genet., 48, 65-69.

Nicholson SE, Willson TA, Farley A, Starr R, Zhang JG, Baca M, Alexander WS, Metcalf D, Hilton DJ and Nicola NA. (1999). EMBO J., 18, 375-385.

Nosaka T, Kawashima T, Misawa K, Ikuta K, Mui AL and Kitamura T. (1999). EMBO J., 18, 4754-4765.

Phelps WC, Yee CL, Munger K and Howley PM. (1988). Cell, 53, 539-547.

Reinstein E, Scheffner M, Oren M, Ciechanover A and Schwartz A. (2000). Oncogene, 19, 5944-5950.

Rottapel R, Ilangumaran S, Neale C, La Rose J, Ho JM, Nguyen MH, Barber D, Dubreuil P and de Sepulveda P. (2002). Oncogene, 21, 4351-4362.
Sakai Y, Kiyotani K, Fukumura M, Asakawa M, Kato A, Shioda T, Yoshida T, Tanaka A, Hasegawa M and Nagai Y. (1999). FEBS Lett., 456, 221-226.

Sasaki A, Yasukawa H, Shouda T, Kitamura T, Dikic I and Yoshimura A. (2000). J. Biol. Chem., 275, 29338-29347.

Selvey LA, Dunn LA, Tindle RW, Park DS and Frazer IH. (1994). J. Gen. Virol., 75 (Part 7), 1647-1653.

Shouda T, Yoshida T, Hanada T, Wakioka T, Oishi M, Miyoshi K, Komiya S, Kosai K, Hanakawa Y, Hashimoto K, Nagata K and Yoshimura A. (2001). J. Clin. Invest., 108, 1781-1788.

Song MM and Shuai K. (1998). J. Biol. Chem., 273, 35056-35062.

Stark GR, Kerr IM, Williams BR, Silverman RH and Schreiber RD. (1998). Annu. Rev. Biochem., 67, 227-264.

Tan TM and Ting RC. (1995). Cancer Res., 55, 4599-4605.

Tomoda K, Kubota Y, Arata Y, Mori S, Maeda M, Tanaka T, Yoshida M, Yoneda-Kato N and Kato JY. (2002). J. Biol. Chem., 277, 2302-2310.

Um SJ, Kim EJ, Hwang ES, Kim SJ, Namkoong SE and Park JS. (2000). Int. J. Cancer, 85, 416-423.

Venturini F, Braspenning J, Homann M, Gissmann L and Sczakiel G. (1999). Nucleic Acids Res., 27, 1585-1592.

von Knebel Doeberitz M, Oltersdorf T, Schwarz E and Gissmann L. (1988). Cancer Res., 48, 3780-3786.

Wang J, Sampath A, Raychaudhuri P and Bagchi S. (2001). Oncogene, 20, 4740-4749.

Watanabe S, Kanda T and Yoshiike K. (1989). J. Virol., 63, 965-969.

Woodworth CD, Lichti U, Simpson S, Evans CH and DiPaolo JA. (1992). Cancer Res., 52, 456-463.

Wu EW, Clemens KE, Heck DV and Munger K. (1993). J. Virol., 67, 2402-2407.

Yasukawa H, Misawa H, Sakamoto H, Masuhara M, Sasaki A, Wakioka T, Ohtsuka S, Imaizumi T, Matsuda T, Ihle JN and Yoshimura A. (1999). EMBO J., 18, 1309-1320.

Yonemitsu Y, Kitson C, Ferrari S, Farley R, Griesenbach U, Judd D, Steel R, Scheid P, Zhu J, Jeffery PK, Kato A, Hasan MK, Nagai Y, Masaki I, Fukumura M, Hasegawa M, Geddes DM and Alton EW. (2000). Nat. Biotechnol., 18, 970-973.

Yonemitsu Y, Alton EW, Komori K, Yoshizumi T, Sugimachi K and Kaneda Y. (1998). Int. J. Oncol., 12, 1277-1285.

Yoshida T, Hanada T, Tokuhisa T, Kosai K, Sata M, Kohara M and Yoshimura A. (2002). J. Exp. Med., 196, 641-653.

Yoshikawa H, Matsubara K, Qian GS, Jackson P, Groopman JD, Manning JE, Harris CC and Herman JG. (2001). Nat. Genet., 28, 29-35.

Zhang JG, Farley A, Nicholson SE, Willson TA, Zugaro LM, Simpson RJ, Moritz RL, Cary D, Richardson R, Hausmann G, Kile BJ, Kent SB, Alexander WS, Metcalf D, Hilton DJ, Nicola NA and Baca M. (1999). Proc. Natl. Acad. Sci. USA, 96, 2071-2076. 